\title{
AVALIAÇÃo DA CAPACITAÇÃO E SATISFAÇÃO DE CHEFES DE COZINHA EM UNIDADES PRODUTORAS DE REFEIÇÕES LOCALIZADAS NA CIDADE DE SÃO PAULO
}

\section{EVALUATION OF TRAINING AND SATISFACTION OF FOOD SERVICE CHEFS IN THE CITY OF SÃO PAULO}

\author{
Aline Veroneze de Mello ${ }^{1}$ \\ Daniele Lima da Cruz ${ }^{1}$ \\ Luana Romão Nogueira ${ }^{1}$ \\ Walleska Luctke Facincani ${ }^{1}$ \\ Andrea Carvalheiro Guerra Matias ${ }^{2}$ \\ Mônica Glória Neumann Spinelli² \\ Edeli Simioni de Abreu ${ }^{2}$
}

Resumo: A qualificação profissional representa o conjunto de habilidades e requisitos necessários para o exercício de uma função, e, para o seu bom desempenho, um fator determinante é o grau de satisfação. Este trabalho teve como objetivo avaliar a formação e nível de satisfação de chefes de cozinha. Foi realizado um estudo transversal por intermédio da aplicação de questionário. A maioria dos chefes de cozinha apresenta ensino médio completo (61\%), e 89\% declararam receber treinamento no próprio estabelecimento de trabalho. Todavia, a maioria dos chefes (66\%) declarou que não há periodicidade pré-definida para o treinamento, sendo realizado "quando necessário". Quanto à motivação para o trabalho, 61\%, declararam "gostar do que faz", sendo que a maioria manifestou satisfação pela profissão (89\%). Dentre as principais dificuldades relatadas para o exercício da função destacaram-se o cansaço físico, o trabalho desgastante, as altas temperaturas do ambiente da cozinha, carregar peso, e funcionários que faltam. A maioria dos chefes de cozinha valoriza o próprio trabalho e sente que é valorizado pelos outros (83\%). Pode-se concluir que, de modo geral, os colaboradores estão satisfeitos com o exercício da função.

Palavras-chave: serviços de alimentação; alimentação coletiva, satisfação no emprego.

Abstract: Professional qualification represents the skill sets and requirements necessary to exercise a function, and for good performance, satisfaction is an important point. This study aimed to assess the level of job satisfaction and capacity of cooks. A cross-sectional study was conducted through a questionnaire. Most cooks had completed high school (61\%), and 89\% reported receiving training in the actual work setting. However, in most cases (66\%), there was no a schedule for training, but it was done "when necessary". As for motivation to work, 61\% said that they "liked what they do", and the majority expressed satisfaction with the profession (89\%). Among the main difficulties reported were the physical exhaustion, the exhausting work, high temperatures in the kitchen, heavy lifting, and absent employees. Most cooks appreciate their work and feel that it is valued by others (83\%). Generally, employees are satisfied with the performance of their work.

Keywords: food services, collective feeding, job satisfaction.

\footnotetext{
1 Graduanda do curso de Nutrição - Universidade Presbiteriana Mackenzie - UPM. E-mails: alinevm3@hotmail.com; danny_ic@msn.com; luanaromaon@hotmail.com; walleskao@hotmail.com.

2 Doutora em Saúde Pública (área de concentração - nutrição) - Universidade de São Paulo - USP. Docente do curso de Nutrição - UPM. E-mails: andrea.matias@mackenzie.br; spinelli@mackenzie.br; edeli.abreu@mackenzie.br.
} 


\section{INTRODUÇÃO}

A qualificação profissional, definida pelo conjunto de habilidades e requisitos que compõem o desenho do cargo ou pelo conhecimento necessário para o desempenho de alguma função (KRAEMER; AGUIAR, 2009), é vista quando um indivíduo possui esse conjunto de habilidades e tem os requisitos necessários para o exercício de suas funções. Está ligada ao sistema de educação formal e, também, aos sistemas de qualificação específicos (educação não formal), oferecidos pelas empresas. Além da qualificação profissional, é importante que sejam desenvolvidas competências que são um conjunto de qualidades ligadas a atitudes, como: iniciativa, criatividade, senso de oportunidade, traços colaborativos para o trabalho coletivo, capacidade de decisão e aptidão para solucionar problemas (FLEURY, 2001; LAUDARES; TOMASI, 2003).

Nesse contexto, os nutricionistas gestores de Unidades Produtoras de Refeições (UPRs) devem sempre planejar, organizar, dirigir, supervisionar e avaliar os serviços de alimentação e nutrição e proporcionar aos funcionários aperfeiçoamento técnico, científico e cultural, também, auxiliá-los em suas funções para, juntos, satisfazerem os usuários com o serviço oferecido (PROENÇA, 2000; BRASIL, 2004).

Por outro lado, o setor encontra desafios, como a baixa qualificação profissional. A formação de qualidade dos profissionais das UPRs é de extrema importância pelo fato da unidade ser dependente da força de trabalho, comprometimento e engajamento de seus funcionários (PROENÇA, 1993; PROENÇA, 1996; BARRETO et al., 2010).

Um fator determinante para o bom desempenho do trabalho é a satisfação. Segundo Spector (2003), a satisfação demonstra como um indivíduo se sente em relação à realização do seu trabalho, suas expectativas pessoais, a relação com os colegas, e com o salário, entre outros aspectos. Além disso, influenciam fatores como a saúde e o sentimento de bem-estar da equipe.

O objetivo deste trabalho foi avaliar o grau de formação e nível de satisfação de chefes de cozinha em UPRs.

\section{METODOLOGIA}

O estudo realizado foi de delineamento transversal, no qual foram entrevistados chefes de cozinha de UPRs de restaurantes comerciais, hospitais, instituições de ensino e empresas, sob sistema de autogestão e concessão (terceirizado), localizadas na cidade de São Paulo. O estudo foi realizado entre os meses de agosto e novembro de 2012.

Como instrumento de coleta de dados, foi elaborado um questionário para levantamento de informações referentes à escolaridade, formação para atuar na área, bem como motivação e satisfação com a função desempenhada.

As variáveis foram analisadas por meio da distribuição da frequência em número e 
porcentagem no programa Microsoft Excel 2010. As questões abertas foram agrupadas de acordo com a metodologia adaptada do Discurso do Sujeito Coletivo, proposta por Lefevre e Lefevre (2003), que consiste na reunião das respostas em apenas um discurso-síntese, escrito em primeira pessoa do singular.

O estudo foi aprovado pela Comissão Interna de Ética em Pesquisa da Universidade Presbiteriana Mackenzie, processo CIEP ${ }^{\circ}$ N001/02/11.

\section{RESULTADOS E DISCUSSÃO}

Ao todo foram entrevistados dezoito chefes de cozinha, sendo $14(78 \%)$ de restaurantes terceirizados e quatro $(22 \%)$ de restaurantes comerciais.

Quanto ao grau de escolaridade, 61\% dos chefes relataram apresentar ensino médio completo. Em estudo de Cavally e Salay (2007), que pesquisaram funcionários de restaurantes do tipo self-service e fast-food localizados em São Paulo e Porto Alegre, foi constatada a prevalência de chefes de cozinha com ensino fundamental completo e incompleto $(59,9 \%)$.

No que concerne à formação específica para atuação na área, $89 \%$ dos chefes declararam receber treinamento no próprio estabelecimento. De acordo com Kraemer e Aguiar (2009), a qualificação da maioria dos chefes de cozinha se dá no decorrer da prática profissional, no próprio local de trabalho, embora a maioria reconheça a importância de um curso profissionalizante, da obtenção de um diploma para futuras promoções ou melhores oportunidades em outras empresas (KRAEMER; AGUIAR, 2009).

Quanto à frequência em que recebem treinamento específico sobre boas práticas de manipulação de alimentos, $66 \%$ dos chefes afirmaram receber treinamento apenas quando necessário, conforme Figura 1. De acordo com a RDC 216 de 15/09/2004 (BRASIL, 2004), os manipuladores de alimentos devem ser supervisionados e capacitados, periodicamente, em higiene pessoal, manipulação higiênica dos alimentos e em doenças transmitidas por alimentos e essa capacitação deve ser comprovada por documentação. Em estudo com manipuladores de uma Unidade de Alimentação e Nutrição de Santa Maria, Rio Grande do Sul, Saccol et al. (2006) verificaram que o conhecimento prévio e grau de aprendizagem dos funcionários de UPRs, sobre o assunto, é pequeno, considerando a necessidade de maior periodicidade em treinamentos. 


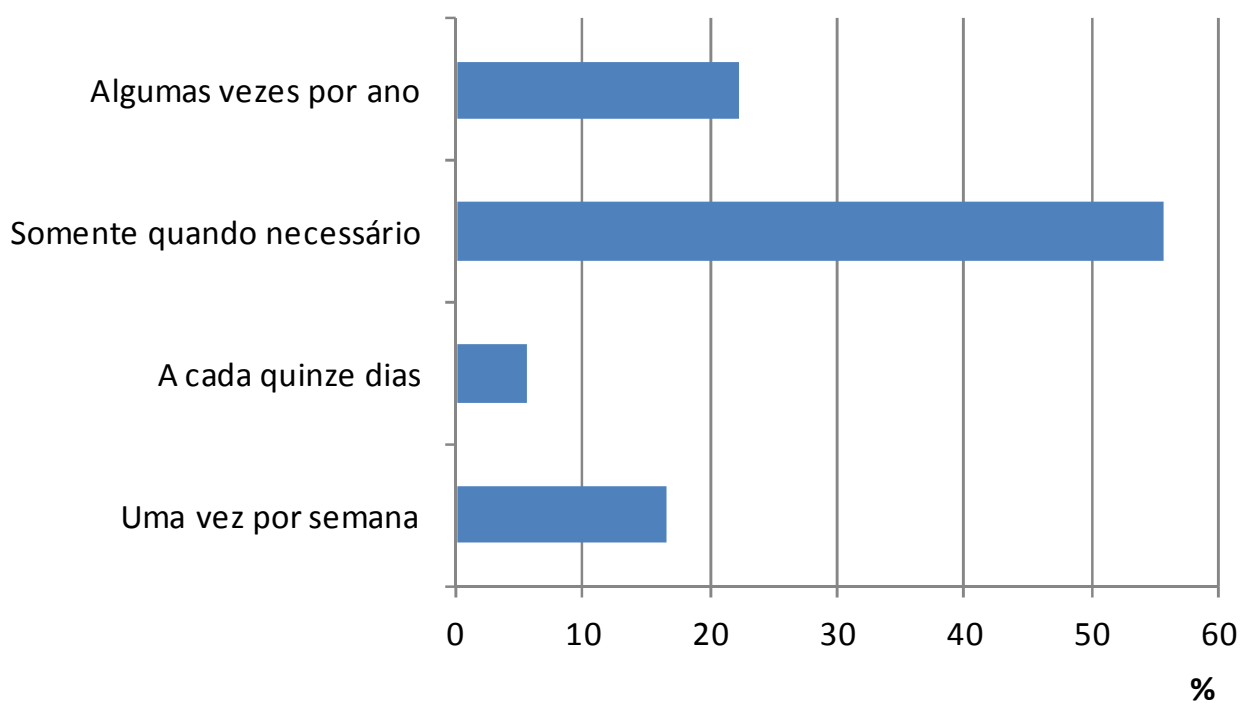

Figura 1 - Distribuição em porcentagem da frequência de treinamentos sobre boas práticas de manipulação de alimentos. São Paulo, 2012.

Referente à motivação para atuar na área, a maioria dos entrevistados (61\%) responderam que a maior motivação é "o gosto" pela área de atuação, em detrimento da remuneração e necessidade (Figura 2). Resultados semelhantes foram encontrados por Maciel (2002), cujo estudo teve como objetivo verificar fatores relacionados à situação de trabalho que poderiam interferir na satisfação de trabalhadores de Unidades de Alimentação e Nutrição. A maioria de seus entrevistados apontou, como um dos itens para a motivação, "gostar do que faz". De acordo com o mesmo autor, a auto realização, quando atendida, produz satisfação.

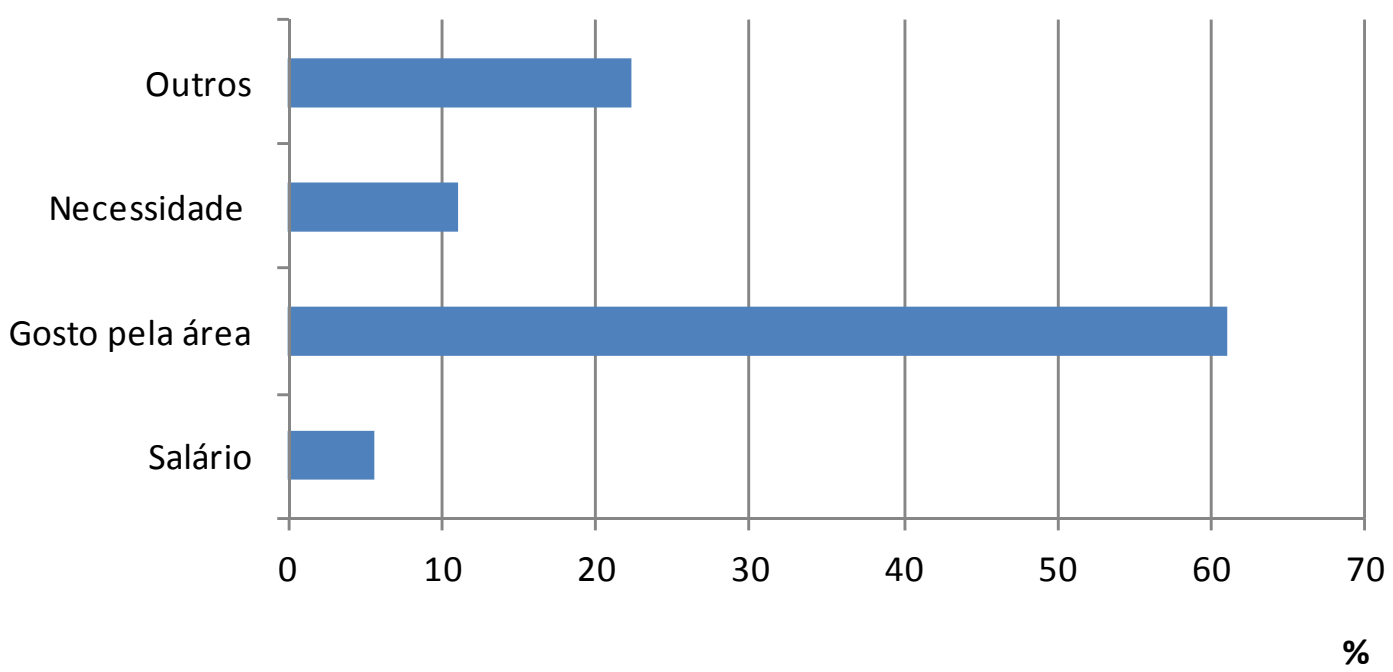

Figura 2 - Distribuição em porcentagem da motivação de chefes de cozinha para o exercício da profissão. São Paulo, 2012. 
Referente à satisfação com a profissão, $89 \%$ dos funcionários relataram estar muito satisfeitos ou satisfeitos com a profissão que exercem, conforme dados apresentados na Figura 3. Resultados semelhantes ao trabalho de Gomez (2006), que estudou uma Unidade de Alimentação e Nutrição localizada no Rio de Janeiro, a respeito do grau de satisfação relacionado ao trabalho e desgaste psicológico no ambiente laboral, em que $79,3 \%$ dos pesquisados se sentiam satisfeitos com a profissão. A formação do conceito de satisfação particular envolve as condições de trabalho, relacionamento com os colegas, carga horária etc. (GOMEZ, 2006). Quanto ao salário, a maioria dos entrevistados deste estudo está insatisfeita, corroborando o estudo de Gomez (2006), no qual a maioria $(58,6 \%)$ de seus entrevistados também considerou o salário pouco satisfatório.

Souza et al. (2013) realizaram um estudo para avaliar a satisfação de funcionários de um hospital privado de pequeno porte, na cidade de Umuarama, Paraná, utilizando uma escala de satisfação específica. Nesse estudo, os funcionários do Serviço de Nutrição e Dietética manifestaram satisfação baixa, intermediária e alta para satisfação com salário, colegas e chefia, respectivamente.

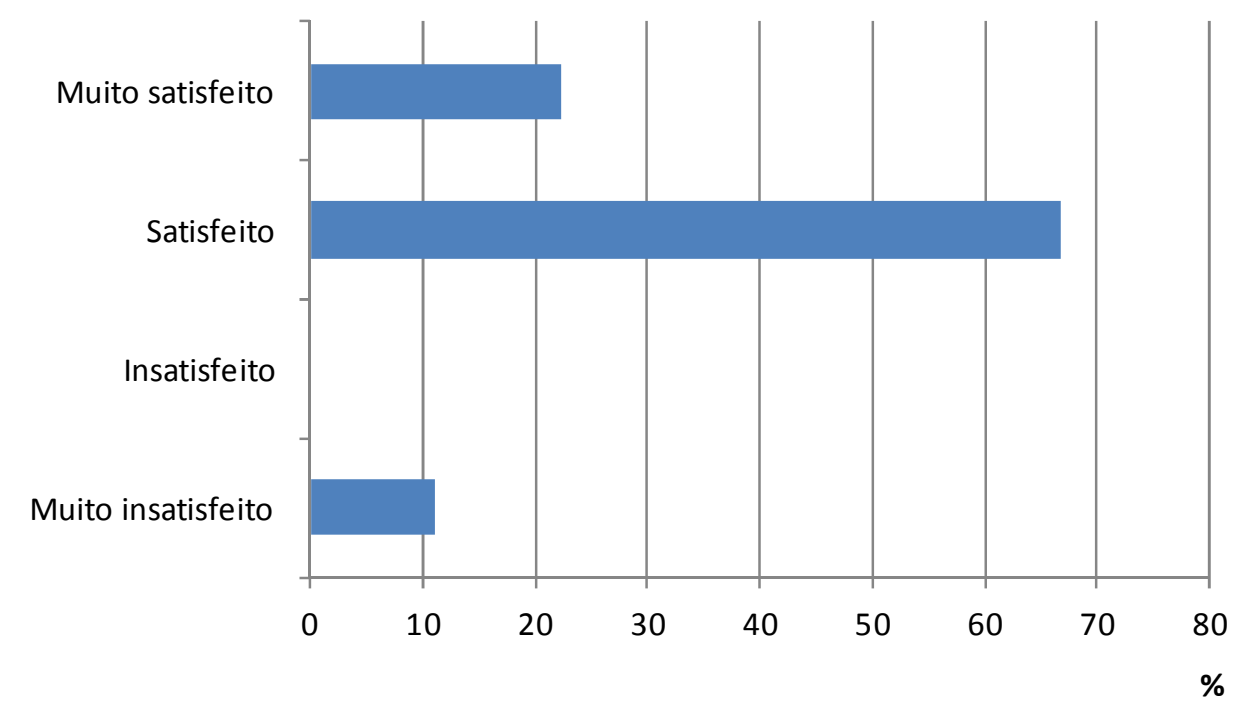

Figura 3 - Distribuição em porcentagem do grau de satisfação de chefes de cozinha pelo exercício da profissão. São Paulo, 2012

As principais dificuldades para atuação na área estão descritas na Tabela 1. 
Tabela 1 - Distribuição, em número e porcentagem, dos discursos-síntese para as principais dificuldades de chefes de cozinha para atuar na área, São Paulo, 2012

\begin{tabular}{lccc}
\hline \multicolumn{1}{c}{ Discurso } & $\mathbf{n}$ & $\%$ \\
\hline "As principais dificuldades da área são: cansaço físico, muita & 12 & 66,7 \\
movimentação, por ficar em pé o dia todo, prejuízo para a coluna, & & \\
trabalho desgastante, em altas temperaturas, carregar peso, & & \\
funcionários que faltam e deixam o trabalho mais desgastante." & & \\
"Outras dificuldades são a formação da equipe, organização, aquisição & 4 & 22,2 \\
de materiais, preparações complicadas, higiene, lidar com pessoas e & & \\
salários baixos." & 2 & 11,1 \\
Entrevistado não relatou sua opinião & 18 & 100,0 \\
Total & & \\
\hline
\end{tabular}

Metade dos chefes de cozinha relatou que o tempo gasto com o transporte para chegar ao trabalho é de duas horas. Bertin et al. (2009), ao avaliarem um Serviço de Nutrição e Dietética de um hospital público de ensino na cidade de São Paulo, com quinze funcionárias, observaram que todas levavam mais de noventa minutos para se deslocarem de casa ao trabalho.

Quanto ao tipo de função, $72 \%$ relataram realizar outras atividades, além das suas próprias, resultado semelhante ao de Maciel (2002), que observou o mesmo para $63 \%$ dos manipuladores. Segundo esse autor, normalmente os funcionários não desgostam de exercer outras atividades, pois isso representa uma quebra na rotina e um sinal de confiança da parte da gerência.

Referente à opinião que os funcionários têm sobre a valorização de seus serviços por parte de outras pessoas, os discursos estão expostos na Tabela 2:

Tabela 2 - Distribuição, em número e porcentagem, dos discursos-síntese quanto à valorização de seus serviços, São Paulo, 2012

\begin{tabular}{lcc}
\hline \multicolumn{1}{c}{ Discurso } & $\mathbf{n}$ & $\%$ \\
\hline "A alimentação é essencial à saúde, todos se alimentam na empresa, & 15 & 83.3 \\
agrega valor, valoriza as pessoas, é importante para a equipe e é valorizado & & \\
pela empresa, com aumento de salário e mudança de cargo. O trabalho é & & \\
realizado com amor e torna a comida melhor, com beleza e sabor dos & & \\
pratos, recebem elogios." & 2 & 11.1 \\
"É importante, mas não recebem elogios." & 1 & 5.6 \\
Entrevistado não relatou sua opinião & 18 & 100.0 \\
Total &
\end{tabular}

Dos pesquisados, 72\% declararam receber horas extras. De acordo com Lemos (1999), uma jornada de trabalho extensiva, ou seja, com mais de oito horas diárias, pode causar improdutividade, devido à fadiga muscular, expondo o trabalhador a risco. O trabalho por doze horas consecutivas pode acometer o indivíduo a doenças ocupacionais. 
A maioria dos entrevistados relatou ter um bom relacionamento com a chefia e com os outros funcionários, resultado compatível com o trabalho de Oliveira et al. (2010), que analisaram uma Unidade de Alimentação e Nutrição de uma Escola Agrotécnica Federal, no estado de Minas Gerais, tendo como participantes colaboradores, fornecedores e clientes, sendo que a maioria atribuiu os conceitos "satisfeito" e "muito satisfeito" às relações com os colegas e superiores.

\section{CONCLUSÃO}

A maioria dos entrevistados apresenta ensino médio e declarou receber treinamento específico para a função no próprio local de trabalho. No entanto, esse treinamento, na maior parte dos casos, é esporádico, "apenas quando necessário". A principal motivação para atuação na área foi "o gosto" pela mesma, concomitante ao elevado grau de satisfação pelo exercício da profissão. Com relação às dificuldades para o exercício da função, destacaramse o cansaço físico, o trabalho desgastante, as altas temperaturas, carregar peso, e funcionários que faltam. Observou-se que os chefes de cozinha valorizam o próprio trabalho e se sentem valorizados pelos outros.

\section{REFERÊNCIAS BIBLIOGRÁFICAS}

BERTIN, C. H. F. et al. O trabalho sob a ótica das manipuladoras de alimentos de uma unidade hospitalar. Campinas, Rev. Nutri., Campinas, v. 22, n. 5, p. 643-652, 2009.

BRASIL. AGENCIA NACIONAL DE VIGILANCIA SANITÁRIA (ANVISA). Resolução - RDC 216 de 15 de setembro de 2004. Diário Oficial da União, Brasília, 2004.

CAVALLI, S. B.; SALAY, E. Gestão de pessoas em unidades produtoras de refeições comerciais e a segurança alimentar. Rev. Nutri., Campinas, v. 20, n. 6, p. 657-667, 2007.

FLEURY, M. T. L. Construindo o conceito de competência. Rev. Adm. Contemp., Curitiba, v. 5, p. 183-96, 2001.

GOMEZ, F. S. Avaliação da satisfação e estresse no trabalho entre funcionários de uma Unidade de Alimentação e Nutrição no Rio de Janeiro. Ceres, Rio de Janeiro, v. 1, n. 1, p. 2642, 2006.

KRAEMER, F. B.; AGUIAR, O. B. Gestão de competências e qualificação profissional no segmento da alimentação coletiva. Rev Nutri., Campinas, v. 22, n. 5, p. 609-619, 2009.

LAUDARES, J. B.; TOMASI, A. O técnico de escolaridade média no setor produtivo: seu novo lugar e suas competências. Educ. Soc., Campinas, v. 24, n. 85, p. 1237-1256, 2003.

LEFEVRE, F.; LEFEVRE, A. M. C. O discurso do sujeito coletivo: um novo enfoque em pesquisa qualitativa. Caxias do Sul: Educs, 2003. 
LEMOS, M. P. Contribuições da ergonomia na melhoria da qualidade higiênico-sanitária de refeições coletivas: um estudo de caso. Florianópolis, 1999. 116f: Dissertação (Mestrado) - Universidade Federal de Santa Catarina, Centro Tecnológico, Florianópolis, 1999.

MACIEL, T. R. S. Fatores interferentes na satisfação dos trabalhadores de uma unidade de alimentação e nutrição hospitalar. Florianópolis, 2002. 58f. Dissertação (Mestrado em Engenharia de Produção). Universidade Federal de Santa Catarina, Centro Tecnológico, Florianópolis, 2002.

OLIVEIRA, M. C. F. et al. Visão global da gestão de uma Unidade de Alimentação e Nutrição Institucional. Nutrire., São Paulo, v. 35, n. 3, p. 115-131, dez. 2010.

PROENÇA, R. P. C. Aspectos organizacionais e inovação tecnológica em processos de transferência de tecnologia: uma abordagem antropotecnológica no setor de alimentação coletiva. Florianópolis. 1996, 306f. Tese (Doutorado em Engenharia de Produção) Universidade Federal de Santa Catarina, Centro Tecnológico, Florianópolis, 1996.

Ergonomia e organização do trabalho em projetos industriais: uma proposta no setor de alimentação coletiva. Florianópolis, 1993. 143f. Dissertação (Mestrado em Engenharia de Produção) - Universidade Federal de Santa Catarina, Centro Tecnológico, Florianópolis, 1993.

Inovação tecnológica na produção de alimentação coletiva. 2. ed. Florianópolis: Insular; 2000.

SACCOL, A. L. F. et al. Importância de treinamento de manipuladores em boas práticas. Disc. Scientia. Série: Ciências da Saúde., Santa Maria, v. 7, n. 1, p. 91-99, 2006.

SPECTOR, P. E. Psicologia nas Organizações. São Paulo: Saraiva, 2003.

SOUZA, I. D. S.; RECHE, P. M.; SACHUCK, M. I. O comprometimento organizacional e a satisfação no trabalho em funcionários de um hospital da cidade de Umuarama - PR. Qualit@s Revista Eletrônica, João Pessoa, v. 14, n. 1, 2013. 